\title{
The Role of the Occipital Artery in the Diagnosis of Intracranial Dural Arteriovenous Fistula Using Duplex Sonography
}

\author{
B.-L. Tee. L.-K. Tsai, C.-C. Lai, S.-C. Tang, Y.-A. Chen, C.-L. Chen, and J.-S. Jeng
}

\begin{abstract}
BACKGROUND AND PURPOSE: The occipital artery is usually a main feeding artery of an intracranial dural arteriovenous fistula. The aim of this study was to establish the role of the OA in the diagnosis of DAVFs by using duplex sonography.
\end{abstract}

MATERIALS AND METHODS: We first compared the clinical features between patients with DAVFs having and not having the OA as one of feeding arteries in 181 consecutive patients with DAVFs. Second, we investigated the OA by using duplex sonography in 60 control subjects to test the accessibility. Finally, we studied 24 DAVF and 60 non-DAVF patients to validate the diagnostic performances of duplex sonography. Hemodynamic parameters, including the resistance index and flow velocity, were analyzed.

RESULTS: Half of the DAVFs (51\%) had the OA as one of feeding arteries. DAVFs with the OA as one of the feeders were more likely located at noncavernous sinuses; to belong to types IIb, Ila $+\mathrm{b}$, III, IV, or V; and to be associated with aggressive manifestations compared with DAVFs without the OA as a feeder $(P<.05)$. Accessibility of the OA by using duplex sonography was $100 \%$. The resistance index was lower and flow velocity was higher in the OA among patients with DAVFs compared with control subjects $(P<.001)$. An OA resistance index $<0.76$ yielded a sensitivity and specificity of $96 \%$ and $97 \%$, respectively, for the diagnosis of a DAVF.

CONCLUSIONS: The OA resistance index can be used to screen for DAVFs having the OA as one of feeding arteries, and this kind of DAVF was usually associated with nonbenign clinical courses.

ABBREVIATIONS: $\mathrm{CCA}=$ common carotid artery; $\mathrm{Cl}=$ confidence interval; $\mathrm{DAVF}=$ dural arteriovenous fistula; $\mathrm{ECA}=$ external carotid artery; $\mathrm{OA}=$ occipital artery

$D^{2}$ ural arteriovenous fistula refers to pathologic vascular channels that are located within the dura mater. ${ }^{1-3}$ The clinical course of DAVFs varies greatly, ranging from spontaneous regression to pulsatile tinnitus and severely debilitating ischemic events to even lifethreatening intracranial hemorrhage. ${ }^{4,5}$ Because catheterized embolization therapy can eliminate DAVFs, ${ }^{6}$ early diagnosis and management of DAVFs may prevent the occurrence of stroke. Cerebral conventional angiography is a standard diagnostic tool for DAVFs, and head CT and MR imaging have a diagnostic sensitivity of $>80 \% \cdot{ }^{3,4,7}$ However, their invasiveness and high cost are disadvantages for DAVF screening. Therefore, a simple and convenient

Received May 17, 2012; accepted after revision June 17.

From the Departments of Neurology and Stroke Centre (B.-L.T., L.-K.T., C.-C.L. S.-C.T., Y.-A.C., C.-L.C., J.-S.J.), National Taiwan University Hospital and National Taiwan University College of Medicine, Taipei, Taiwan; Department of Neurology (B.-L.T.), En Chu Kong Hospital, New Taipei City, Taiwan; and Department of Neurology (L.-K.T.), National Taiwan University Hospital, Yun-Lin Branch, Yun-Lin, Taiwan.

Please address correspondence to Li-Kai Tsai, MD, PhD, Department of Neurology, National Taiwan University Hospital, No. 7 Chung-Shan South Rd, Taipei 100, Taiwan; e-mail address: milikai@ntuh.gov.tw

http://dx.doi.org/10.3174/ajnr.A3273 screening method for DAVF is still needed, especially for screening asymptomatic and mildly symptomatic patients who may possibly develop aggressive manifestations in the future.

Diagnosis of a DAVF by sonography mainly depends on hemodynamic changes in the feeding arteries. ${ }^{8-12}$ DAVFs may result in reduced flow resistance and increased flow velocity in the carotid arteries because of the direct shunts from their branches to the sinuses or veins. ${ }^{13}$ In our previous studies by using carotid duplex sonography, $75 \%$ of patients with DAVFs had a low resistance index in the external carotid arteries. ${ }^{8,9}$ To improve the detection rate for DAVFs by using duplex sonography, 1 small study further tried to analyze hemodynamic changes in branches of the ECAs, such as the occipital artery, and demonstrated a positive predictive value of $100 \% .{ }^{11}$ This is because hemodynamic abnormalities are more likely detected in feeding arteries closer to the DAVF. The OA normally arises from the ECA and travels to the occipital groove along the medial border of the mastoid process. ${ }^{14}$ Because the OA is one of the common feeding arteries for DAVFs and can be easily studied by using duplex sonography, ${ }^{11}$ evaluation of abnormal hemodynamic changes in the OA by using sonography is a potential screening method for detecting DAVFs. 
To establish the important role of the OA in the diagnosis of DAVFs, we first studied a large cohort of patients with DAVFs and analyzed the prevalence and characteristics of DAVFs with the OA as one of the feeding arteries. We then investigated the hemodynamics in the OA in both patients with DAVFs and control subjects to test the accessibility of the OA on duplex sonography and to validate the diagnostic performance by using OA-related duplex parameters.

\section{MATERIALS AND METHODS Study Design and Patients}

The present study comprised 3 parts: 1) comparison of DAVFs with and without the OA as one of the feeders, 2) accessibility of the OA on duplex sonography, and 3) diagnosis of DAVFs by using duplex sonography on the OA.

In the first part of the study, a total of 181 consecutive patients who had been admitted to the National Taiwan University Hospital from May 1995 through August 2010 and were diagnosed as having a DAVF were enrolled in the study. The patients' records were reviewed retrospectively, and demographic data and clinical manifestations were recorded. The diagnosis of DAVF was confirmed by cerebral conventional angiography. Patients were classified into 2 groups: having the OA as one of the feeding arteries (group 1) and not having the OA as one of feeding arteries (group 2). The demographic data, aggressiveness of clinical presentation, and characteristics of the DAVFs were compared between the 2 groups.

In the second part of study, 60 adult control subjects ( 31 men; age, $58 \pm 13$ years) without tinnitus, ocular discomfort, or symptoms of cerebrovascular disorders were included from September 2008 through March 2011. Bilateral OAs and carotid arteries were assessed by using duplex sonography.

In the third part of study, data of 24 patients with DAVFs in group 1 (from April 2002 through August 2010) who had undergone duplex sonographic studies on both the occipital and carotid arteries were initially compared with data obtained from 60 control subjects. We further enrolled 60 patients without DAVF (non-DAVF) (38 men; age, $69 \pm 12$ years) from October 2011 to May 2012. The hemodynamics were then investigated in the OA and carotid arteries in 24 patients with DAVFs and 60 non-DAVF patients to validate the diagnostic performance by using duplex parameters. These non-DAVF patients all underwent either head MR imaging (with or without contrast medium injection) or CT (with and without contrast medium injection), and there was no evidence for DAVFs such as abnormal flow void, venous infarct, sinus thrombosis, or engorgement of cortical veins or extracranial arteries. ${ }^{3,7}$ The clinical diagnosis of these patients included cerebral infarct $(n=31)$, arterial stenosis $(n=9)$, transient ischemic attack $(n=4)$, vertigo $(n=4)$, malignancy $(n=3)$, parkinsonism $(n=2)$, dementia $(n=2)$, aneurysm $(n=2)$, head injury $(n=1)$, intracerebral hemorrhage $(n=1)$, and encephalitis $(n=1)$. Each subject underwent a complete duplex sonographic study with the same machines as those used for patients with DAVF.

\section{Characteristics of DAVFs}

The location of the DAVF was classified as the cavernous sinus, large sinus, or "other" as previously described. ${ }^{5,15}$ The location "large sinus" included transverse, sigmoid, and superior sagittal sinuses. "Other" locations were those other than the cavernous and large sinuses. We also classified DAVFs into 5 types according to the venous drainage pattern following the method of Cognard et $\mathrm{al}^{4}$ : type I, drainage into a sinus with normal antegrade flow; type II, drainage into a sinus with retrograde venous drainage into the sinus (type IIa), with retrograde venous drainage into a cortical vein (type IIb), or with retrograde venous drainage into the sinus and cortical vein (type IIa $+\mathrm{b}$ ); type III, drainage directly into a cortical vein without venous ectasia; type IV, drainage into a cortical vein with venous ectasia $>5 \mathrm{~mm}$ in diameter or 3 times larger than the diameter of the draining vein; and type $V$, drainage into the spinal perimedullary veins.

On the basis of the clinical manifestations, DAVFs were divided into "aggressive" and "nonaggressive" subgroups, similar to those in previous reports. ${ }^{4,15,16}$ The aggressive group included patients with intracranial hemorrhage, venous infarction, seizure, altered mental status, and intracranial hypertension. The nonaggressive group included patients who had tinnitus, ocular symptoms not related to intracranial hypertension, and cranial neuropathy.

\section{Duplex Sonography}

The duplex sonography studies were performed with an HP 4500 system (Hewlett-Packard Company, Palo Alto, California) containing a 3- to 11-MHz real-time B-mode imaging transducer and a 3.6-MHz pulsed Doppler transducer, or a VST Master system (Diasonics, Tirat Carmel, Israel) containing a 10-MHz real-time B-mode imaging transducer and a 6-MHz pulsed Doppler transducer. The OA was examined by placing a sonography probe posterior to the mastoid process at an angle of $15^{\circ}-30^{\circ}$ from the vertical line where the OA emerges to ascend to the occipital sulcus. The angle of insonation for measurement of hemodynamics in the OAs and carotid arteries was $<60^{\circ}$. Resistance index; peak systolic velocity; and end diastolic velocity of the common carotid artery, proximal ECA, and OA were measured once automatically by sonographic machines. The resistance index, which represents flow resistance, was defined as (peak systolic velocity - end diastolic velocity)/peak systolic velocity. ${ }^{17}$ Duplex sonography was performed by sonographic experts (C.-C.L., Y.-A.C., B.-L.T., and L.-K.T.) who were blinded to clinical characteristics and imaging data of the patients. Duplex results from bilateral arteries were obtained, and we analyzed the data from the side with a greater change in values in patients with DAVFs. In Part 2 of the study, the data collected from the right and left arteries of control subjects were averaged and used as the cutoff points for abnormal criteria at the fifth and 95th percentile values. Nine duplex parameters were further validated for diagnosis of DAVFs, including resistance index; peak systolic velocity; and end diastolic velocity in the OA, ECA, and CCA.

\section{Statistical Analysis}

The Fisher exact test was used to establish the differences in categoric data between groups. The Student $t$ test was used to determine the differences in age and duplex data. The sensitivity, specificity, positive predictive value, negative predictive value, and accuracy were also determined in each duplex sonography parame- 
Table 1: Patients with dural arteriovenous fistulas with and without an occipital artery as one of the feeders ${ }^{a}$

\begin{tabular}{lccc} 
& \multicolumn{2}{c}{ Dural Arteriovenous Fistulas } & \\
\cline { 2 - 3 } & $\begin{array}{c}\text { With OA } \\
(\boldsymbol{n}=\mathbf{9 2})\end{array}$ & $\begin{array}{c}\text { Without OA } \\
(\boldsymbol{n}=\mathbf{8 9})\end{array}$ & P Value \\
\hline Age (yr) (SD) & $54 \pm 14$ & $56 \pm 16$ & .19 \\
Male (\%) & $44(48)$ & $29(33)$ & .04 \\
Location (\%) & & & \\
Cavernous sinus & $3(3)$ & $60(9)$ & \\
Large sinuses & & $9(10)$ & \\
Other sinuses ${ }^{b}$ & $60(65)$ & $20(23)$ & \\
Type (\%) & $29(32)$ & & \\
I and Ila & & $53(60)$ & \\
IIb, Ila+b, and III & $48(52)$ & $34(38)$ & .001 \\
IV and V & $10(11)$ & $2(2)$ & \\
Aggressiveness (\%) & $36(39)$ & $21(24)$ & \\
\hline
\end{tabular}

a Values are numbers (percentages), except age.

${ }^{\mathrm{b}}$ Large sinuses include the transverse, sigmoid, and superior sagittal sinuses; other sinuses include all locations other than the cavernous sinus and large sinuses.

Table 2: Hemodynamics using duplex sonography in control subjects

\begin{tabular}{cccc}
\hline & Right & Left & $P$ Value \\
\hline OA & & & \\
RI & $0.89 \pm 0.08$ & $0.89 \pm 0.07$ & .81 \\
PSV & $54 \pm 21$ & $52 \pm 22$ & .59 \\
EDV & $6 \pm 5$ & $6 \pm 4$ & .92 \\
ECA & & & \\
RI & $0.87 \pm 0.06$ & $0.85 \pm 0.05$ & .18 \\
PSV & $71 \pm 17$ & $69 \pm 19$ & .42 \\
EDV & $10 \pm 4$ & $10 \pm 4$ & .52 \\
CCA & & & \\
RI & $0.72 \pm 0.06$ & $0.71 \pm 0.07$ & .70 \\
PSV & $75 \pm 17$ & $77 \pm 16$ & .45 \\
EDV & $21 \pm 6$ & $22 \pm 6$ & .46 \\
\hline
\end{tabular}

Note:-EDV indicates end diastolic velocity (centimeters/second); PSV, peak systolic velocity $(\mathrm{cm} / \mathrm{s})$; RI, resistance index.

ter. Two-sided $P$ values $<.05$ were considered to indicate a statistically significant difference. STATA software (Version 7.0; StataCorp, College Station, Texas) was used for the statistical analyses.

\section{RESULTS}

\section{Part 1: Comparison of DAVFs with and without the OA as} One of Feeders

Of the 181 patients (73 men, 40\%) with DAVF, the average age at diagnosis was $55 \pm 15$ years (range, 18-83 years). Fifty-seven patients $(31 \%)$ developed aggressive symptoms. Ninety-two DAVFs (51\%) had the OA as one of the feeding arteries.

Table 1 shows the features of DAVFs with and without the OA as one of the feeders. More men had DAVFs with the OA as one of feeders than women (48\% versus 33\%, $P=.04$ ). Compared with DAVFs without the OA as a feeder, DAVFs with the OA as one of feeders were more likely located at the large sinuses or "other" sinuses (97\% versus 33\%, $P<.001$ ) and belonged to types IIb, IIa $+\mathrm{b}$, III, IV, or $\mathrm{V}(63 \%$ versus $40 \%, P<.001)$. In 118 patients with DAVFs located in the noncavernous sinuses, $89(75 \%)$ had the OA as one of feeders. In 94 patients with DAVFs belonging to types IIb, IIa + b, III, IV, or V, $58(62 \%)$ had the OA as one of feeders. In addition, among 57 patients with noncavernous sinus DAVFs and having pulsatile tinnitus, $53(93 \%)$ had the OA as one of feeding arteries. Compared with DAVFs without the OA as a feeder, DAVFs with the OA as one of
Table 3: Hemodynamics in patients with DAVF and control subjects using duplex sonography

\begin{tabular}{ccccc}
\hline & $\begin{array}{c}\text { DAVF } \\
(\boldsymbol{n}=\mathbf{2 4})\end{array}$ & $\begin{array}{c}\text { Control } \\
(\boldsymbol{n}=60)\end{array}$ & $\boldsymbol{P}$ Value & Ratio $^{\mathbf{a}}$ \\
\hline OA & & & & \\
RI & $0.49 \pm 0.13$ & $0.89 \pm 0.07$ & $<.001$ & 0.53 \\
PSV & $119 \pm 48$ & $53 \pm 19$ & $<.001$ & 2.9 \\
EDV & $64 \pm 30$ & $6 \pm 4$ & $<.001$ & 14.6 \\
ECA & & & & \\
RI & $0.6 \pm 0.09$ & $0.86 \pm 0.05$ & $<.001$ & 0.67 \\
PSV & $112 \pm 31$ & $70 \pm 16$ & $<.001$ & 1.7 \\
EDV & $45 \pm 17$ & $10 \pm 4$ & $<.001$ & 4.9 \\
CCA & & & & \\
RI & $0.62 \pm 0.07$ & $0.72 \pm 0.06$ & $<.001$ & 0.86 \\
PSV & $93 \pm 21$ & $76 \pm 15$ & .007 & 1.2 \\
EDV & $35 \pm 12$ & $22 \pm 6$ & $<.001$ & 1.7 \\
\hline
\end{tabular}

Note:-EDV indicates end diastolic velocity $(\mathrm{cm} / \mathrm{s})$; PSV, peak systolic velocity $(\mathrm{cm} /$ s); RI, resistance index.

${ }^{a}$ Ratio is the data collected from patients with DAVFs versus those of control subjects.

Table 4: Diagnostic performances of duplex parameters for dural arteriovenous fistula

\begin{tabular}{|c|c|c|c|c|c|c|}
\hline & Cutoff & Sensitivity & Specificity & PPV & NPV & Accuracy \\
\hline \multicolumn{7}{|l|}{ OA } \\
\hline RI & 0.76 & 0.96 & 0.97 & 0.92 & 0.98 & 0.96 \\
\hline PSV & 92 & 0.67 & 0.88 & 0.70 & 0.87 & 0.82 \\
\hline EDV & 14 & 0.96 & 0.95 & 0.88 & 0.98 & 0.95 \\
\hline \multicolumn{7}{|l|}{$\mathrm{ECA}$} \\
\hline RI & 0.75 & 0.88 & 0.87 & 0.72 & 0.95 & 0.87 \\
\hline PSV & 103 & 0.58 & 0.58 & 0.36 & 0.78 & 0.58 \\
\hline EDV & 18 & 0.96 & 0.67 & 0.53 & 0.98 & 0.75 \\
\hline \multicolumn{7}{|l|}{ CCA } \\
\hline RI & 0.59 & 0.29 & 0.98 & 0.88 & 0.78 & 0.79 \\
\hline PSV & 107 & 0.25 & 0.87 & 0.43 & 0.74 & 0.69 \\
\hline EDV & 33 & 0.50 & 0.95 & 0.80 & 0.83 & 0.82 \\
\hline
\end{tabular}

Note:-EDV indicates end diastolic velocity (centimeters/second); NPV, negative predictive value; PPV, positive predictive value; PSV, peak systolic velocity $(\mathrm{cm} / \mathrm{s})$; RI, resistance index.

feeders were more likely associated with aggressive presentations (39\% versus $24 \%, P=.03$ ).

\section{Part 2: Accessibility of the OA by Using Duplex Sonography}

The flow in the OA went up and back toward the occipital groove, and the hemodynamics in the OA could be directly investigated by using Doppler sonography. In 120 OAs from 60 control subjects, the accessibility by using duplex sonography to detect the resistance index and flow velocity in OAs was $100 \%$. There was no difference in resistance index, peak systolic velocity, or end diastolic velocity between bilateral OAs (Table 2). The Doppler waveform was similar in the OAs and ECAs; however, the resistance index was higher $(P<$ $.01)$ and peak systolic velocity and end diastolic velocity were significantly lower $(P<.001)$ in OAs than in ECAs and CCAs. When we studied 16 OAs, the intraobserver correlation coefficients were $80 \%$, $75 \%$, and $75 \%$, while the interobserver correlation coefficients were $85 \%, 28 \%$, and $81 \%$ for the resistance index, peak systolic velocity, and end diastolic velocity, respectively.

\section{Part 3: Diagnosis of DAVF by Using Duplex Sonography of the $O A$}

Results of duplex sonography were first compared between 24 patients with DAVFs having the OA as one of feeders and 60 

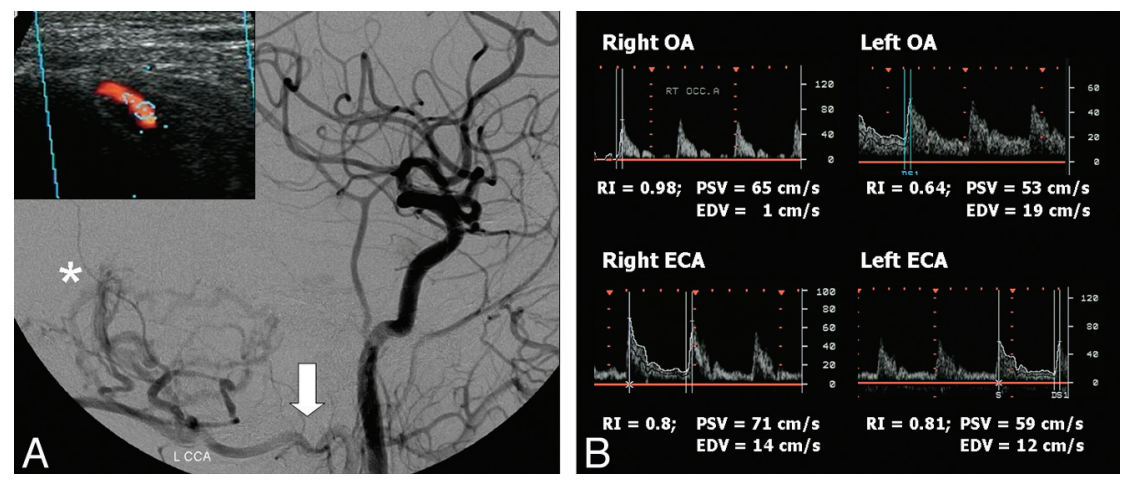

of the OA by using duplex sonography was $100 \%$ and the intraobserver and interobserver variability in the investigation of the OA resistance index was low, with a correlation coefficient of $>80 \%$. In addition, the values of the OA resistance index were abnormally decreased in nearly all DAVFs with the OA as one of feeding arteries. Previous studies have shown that noncavernous sinus location and types IIb/IIa + b, III, IV, and V DAVFs were major determinants of aggressive clinical manifestations. ${ }^{4,15,16}$ In this study, DAVFs with the OA as one of feeders were more likely associated with aggressive manifestations, and the OA was one of the feeding arteries in $75 \%$ of DAVFs located in noncavernous sinuses and in $62 \%$ of

control subjects (Table 3). The resistance index of the OA, ECA, and CCA was significantly lower and the peak systolic velocity and end diastolic velocity were significantly higher in patients with DAVFs than in control subjects $(P<.01)$. To demonstrate the greater change in hemodynamics in the OA than in the ECA or CCA among patients with DAVFs, we further analyzed the differences in sonographic data from the OA, ECA, and CCA between patients with DAVFs and control subjects. When we compared data collected from patients with DAVFs with those of control subjects, the resistance index ratio differences were $0.53,0.67$, and 0.86 in the OA, ECA, and CCA, respectively. In addition, the peak systolic velocity ratio differences were $2.9,1.7$, and 1.2 , respectively, and the end diastolic velocity ratio differences were 14.6, 4.9, and 1.7, respectively, in the OA, ECA, and CCA (Table 3).

By using norm values established from the control subjects, the diagnostic performances of all 9 parameters were determined in 24 DAVF and 60 non-DAVF patients (Table 4). Among all parameters, the resistance index of the OA yielded the best diagnostic performance with a sensitivity, specificity, positive predictive value, negative predictive value, and accuracy of 96, 97, 92, 98, and 96, respectively. For DAVFs having the OA as one of the feeding arteries, the OA resistance index from 23 of 24 patients (96\%) was lower than the cutoff value of 0.76 . On the other hand, the OA resistance index from only 2 of 60 non-DAVF patients (3\%) was lower than 0.76 . The clinical diagnosis of these 2 patients was stroke and dementia, respectively. Notably, when the resistance index of the ECA was lower than the cutoff value of 0.75 in patients with DAVFs, the resistance index of the OA was also abnormally low. However, the ECA resistance indices in 2 patients with DAVFs were normal, while the values of the OA resistance index were still obviously reduced ( 0.64 and 0.3 , respectively; Fig $1)$.

\section{DISCUSSION}

Early diagnosis and treatment of DAVFs can prevent stroke. Because the $\mathrm{OA}$ is usually one of the major feeding arteries of DAVFs, ${ }^{11,18}$ abnormal hemodynamics in the OA might be useful for screening of DAVFs. Here, we demonstrated that accessibility
DAVFs with types IIb, IIa $+b$, III, IV, and V patterns of venous drainage. Taken together, these results suggest that the resistance index of the OA can be used to screen for DAVFs having the OA as one of feeding arteries and this kind of DAVF is usually associated with nonbenign clinical courses.

The OA normally arises from the ECA and travels along the medial border of the mastoid process to the occipital regions. ${ }^{14}$ Therefore, most DAVFs located at the transverse-sigmoid sinuses or brain base had feeding arteries including the OA. In addition, DAVFs with feeding arteries including the OA were usually associated with fulminant cortical venous drainage patterns. There is an abundance of anastomoses between the OA and meningeal arteries at the intracranial posterior fossa. ${ }^{14}$ The OA may thus potentially supply DAVFs via multiple shunts, subsequently resulting in venous hypertension and retrograde cortical venous drainage patterns. DAVFs in men were likely to have the OA as one of feeding arteries, though the reason for this is unknown.

Pulsatile tinnitus is a common initial symptom in patients with DAVFs and is associated with the DAVF having the OA as a feeder because the OA travels close to the ear. ${ }^{18}$ In our patients with pulsatile tinnitus and a noncavernous sinus DAVF, the OA was one of feeding arteries in $93 \%$ of the cases. One study showed that all 17 patients with DAVFs with pulsatile tinnitus had abnormal hemodynamics in the OA by using duplex sonography. ${ }^{11}$ Another study also demonstrated that the most frequent Doppler finding in 16 patients with DAVFs with pulsatile tinnitus was a low-resistance flow profile with increased flow velocities in ECAs and OAs. ${ }^{10}$ In our patients with pulsatile tinnitus and having the $\mathrm{OA}$ as a feeder of the DAVF, all the OA resistance indices were also lower than 0.76. Pulsatile tinnitus in patients with DAVFs may indicate high-flow velocity and turbulent flow around the arteriovenous shunts. ${ }^{18}$ Therefore, the ability to detect the hemodynamic changes by duplex sonography is thus higher. Given a strong relationship between pulsatile tinnitus and DAVFs that have the $\mathrm{OA}$ as a feeder and the high diagnostic performance of sonography in this patient group, using duplex sonography to study flow patterns in the OA is a good method to screen for DAVFs in patients with pulsatile tinnitus.

Compared with arteries supplying brain tissue or facial organs, 
arteries supplying muscles usually have higher flow resistance and lower flow velocity. ${ }^{17}$ The CCA branches into the ECA and ICA (supplying brain tissue), and the ECA further branches into the OA (essentially a muscular branch) and other arteries (supplying head and neck tissues including organs). Therefore, the resistance index is higher, and the peak systolic velocity and end diastolic velocity are lower in the OA than in the ECA and CCA under normal conditions. In contrast, because the hemodynamics of feeding arteries closer to a DAVF would more likely be influenced by the shunts, the OA in patients with DAVFs exhibited a lower resistance index and higher peak systolic velocity and end diastolic velocity than the ECA or CCA. As a result, the differences in resistance index and flow velocity between patients with DAVFs and healthy subjects were more prominent in the OA than in the ECA and CCA. In clinical practice, the resistance index and flow velocity in the ECA can be used to screen for DAVFs. ${ }^{8,12,13}$ However, the hemodynamic changes are sometimes equivocal in the ECA, and physicians might be hesitant to do further work-up in these borderline cases. In such a situation, additional study of the resistance index and flow velocity in the OA would be helpful (ie, patients with a low resistance index and high peak systolic velocity and end diastolic velocity values in the OA should undergo further neuroimaging studies).

Parameters of the OA resistance index yielded a high sensitivity, specificity, positive predictive value, and negative predictive value that resulted in nearly $100 \%$ diagnostic accuracy for diagnosis of DAVFs when the OA was one of feeding arteries. Because duplex sonography has been widely used to evaluate the severity of atherosclerosis in the carotid arteries, ${ }^{19}$ simultaneous analysis of the OA resistance index during routine carotid duplex sonography study may detect occult DAVFs in some patients.

Although duplex sonography is a convenient, inexpensive, and noninvasive screening tool for DAVFs, angiography still is the standard diagnostic study, which reveals the locations and venous drainage patterns of DAVFs. ${ }^{2,4}$ In our series, only $5 \%$ of DAVFs located at the cavernous sinus were supplied from the OA, which impeded the diagnosis of this kind of DAVF using duplex sonography of the OA. However, DAVFs located at the cavernous sinus almost always have a benign course, ${ }^{4,20}$ and screening focusing on DAVFs in locations other than the cavernous sinus is more clinically important. In addition, 25\% of noncavernous sinus DAVFs did not have the OA as a feeder; thus, the OA cannot totally replace the ECA in screening for DAVFs by using duplex sonography. In this study, only 24 of 92 patients (26\%) with DAVFs having the $\mathrm{OA}$ as one of feeding arteries underwent duplex sonography for further analysis. There was no difference in sex, clinical aggressiveness, or DAVF characteristics between patients with DAVFs with or without duplex sonography studies; therefore, the possibility of patient selection bias can be ignored. Conventional angiography was not performed in 60 non-DAVF patients; thus, the possibility of DAVFs cannot be totally ruled out. However, if one takes into account the low prevalence of DAVFs and the absence of evidence for DAVFs by using MR imaging or CT in these patients, the chances of a DAVF in the non-DAVF group is highly unlikely.

\section{CONCLUSIONS}

Hemodynamic parameters of the OA provide useful information for the diagnosis of DAVFs. Patients planning to undergo routine carotid duplex sonography for any reason or those having DAVFrelated symptoms (eg, pulsatile tinnitus) should also undergo duplex studies of the OAs. If the OA resistance index is $<0.76$, then neuroimaging such as CT, MR imaging, or cerebral conventional angiography should be performed to rule out the possibility of a DAVF. Using the above diagnostic protocol could identify $75 \%$ of noncavernous sinus DAVFs and $62 \%$ of DAVFs with fulminant venous drainage patterns.

\section{REFERENCES}

1. Aminoff MJ. Vascular anomalies in the intracranial dura mater. Brain 1973;96:601-12

2. Gupta A, Periakaruppan A. Intracranial dural arteriovenous fistulas: a review. Indian J Radiol Imaging 2009;19:43-48

3. Chung SJ, Kim JS, Kim JC, et al. Intracranial dural arteriovenous fistulas: analysis of 60 patients. Cerebrovasc Dis 2002;13:79-88

4. Cognard C, Gobin YP, Pierot L, et al. Cerebral dural arteriovenous fistulas: clinical and angiographic correlation with a revised classification of venous drainage. Radiology 1995;194:671-80

5. Yeh SJ, Tsai LK, Liu HM, et al. Ischemic stroke in patients with intracranial dural arteriovenous fistulas. J Formos Med Assoc 2011; 110:299-305

6. Narayanan S. Endovascular management of intracranial dural arteriovenous fistulas. Neurol Clin 2010;28:899-911

7. Lee CW, Huang A, Wang $\mathrm{YH}$, et al. Intracranial dural arteriovenous fistulas: diagnosis and evaluation with 64-detector row CT angiography. Radiology 2010;256:219-28

8. Tsai LK, Jeng JS, Wang HJ, et al. Diagnosis of intracranial dural arteriovenous fistulas by carotid duplex sonography. J Ultrasound Med 2004;23:785-91

9. Tsai LK, Liu HM, Lu CJ, et al. Carotid duplex sonography in the follow-up of intracranial dural arteriovenous fistulae. AJNR Am J Neuroradiol 2005;26:625-29

10. Waldvogel D, Mattle HP, Sturzenegger M, et al. Pulsatile tinnitus: a review of 84 patients. J Neurol 1998;245:137-42

11. Arning C, Grzyska U, Lachenmayer L. Duplex ultrasound of external carotid artery branches for the detection of dural arteriovenous fistulae [in German]. Rofo 2005;17:236-41

12. Tsai LK, Yeh SJ, Chen YC, et al. Screen for intracranial dural arteriovenous fistulae with carotid duplex sonography. J Neurol Neurosurg Psychiatry 2009;80:1225-29

13. Tsai LK, Jeng SJ, Yip PK. Ultrasonography in intracranial dural arteriovenous fistula. J Med Ultrasound 2008;22:157-64

14. Lasjaunias P, Theron J, Moret J. The occipital artery. Neuroradiology 1978;15:31-37

15. Tsai LK, Jeng JS, Liu HM, et al. Intracranial dural arteriovenous fistulas with or without cerebral sinus thrombosis: analysis of 69 patients. J Neurol Neurosurg Psychiatry 2004;75:1639-41

16. Awad IA, Little JR, Akrawi WP, et al. Intracranial dural arteriovenous malformations: factors predisposing to an aggressive neurological course. J Neurosurg 1990;72:839-50

17. Hennerici MG, Neuerburg-Heusler D. Vascular Diagnosis with Ultrasound: Clinical Reference with Case Studies. 2nd ed. New York: Thieme Medical Publishers; 2006:351-52

18. Yeh SJ, Tsai LK, Jeng JS. Clinical and carotid ultrasonographic features of intracranial dural arteriovenous fistulae in patients with and without pulsatile tinnitus. J Neuroimaging 2010;20:354-58

19. U.S. Preventive Services Task Force. Screening for carotid artery stenosis: U.S. Preventive Services Task Force Recommendation Statement. Ann Intern Med 2007;147:854-59

20. Barrow DL, Spector RH, Braun IF, et al. Classification and treatment of spontaneous carotid-cavernous sinus fistulas. J Neurosurg 1985; 62:248-56

AJNR Am J Neuroradiol 34:547-51 Mar 2013 www.ajnr.org 551 